James A. Matthewson

is the founder of Trust Digital,

a digital marketing

consultancy. James is a

published author on e-business

and e-marketing and is the

course tutor of the Institute of

Direct Marketing's Certificate

in Digital Marketing. He also

speaks at conferences all over

the world on online advertising.
Keywords: impressions, CpM, behavioural targeting, ROI, rules-based, optimisation, media yield, passback, pass-sideways, predictive, $\mathrm{CpA}$, progressive, realtime decisioning
James A. Matthewson

Trust Digital

London, UK

Tel: +4407734314540

E-mail: james@trustdigital.co.uk

\section{Behavioural targeting: Can online advertising deliver in 2006?}

\author{
James A. Matthewson
}

Received (in revised form): 30 September 2005

\begin{abstract}
Online marketing strategy today comprises a wide range of tactics, including search, e-mail, viral, blogs and advertising. But it is on the last of these activities, online advertising, that much of the debate around the effectiveness and contribution to the overall marketing plan continues to focus. In the same way as in the fashion and music industries, online marketing is a victim of trends: online advertising, once very much out of fashion, is now back in fashion. Part of the reason for its renaissance is that its users have learnt to apply traditional DM and database marketing methodologies to its business model. As a result the concept of 'behavioural targeting' is becoming a new weapon in the banner advertiser's arsenal, a weapon which wields great power to deliver better click and conversion rates and reduce the biggest issue of all in the media spend, wastage.

Behavioural targeting is now helping to challenge the entrenched ROI models of effective $\mathrm{CpA}$, and to deliver enhanced long-term value.
\end{abstract}

\section{Introduction - What is behavioural targeting?}

In 2005 one of the greatest areas of predicted growth in digital marketing was in online advertising. The Interactive Advertising Bureau predicts online advertising to be worth around $£ 650 \mathrm{~m}$.

Yet it is often difficult to talk to online marketing professionals about web advertising without their complaining about low click-through rates (CTRs), high media costs and poor ROI. Online advertising is clearly a medium which is perceived to suffer serious problems.

A central problem, which underlies CTRs and ROI, is relevance. This paper addresses strategic opportunities to improve relevance. The objective of these tools is to deliver an experience analogous to that of the reader of a morning newspaper who only sees advertising specifically relevant to himself or herself. This would be equivalent to the publisher being able to adapt the advertising in that newspaper in each copy to suit the interests of individual readers even before the paper is printed

Consider now the situation in respect of online advertising. One of the reasons why online advertising has not been effective in the past is that the banner and pop-up advertisements which customers see are far too generalised and unspecific to their individual interests and requirements. While customer data have existed in a form whereby some segmentation can be applied online, most website publishers have not been able to act 
on those data on account of the split-second decisioning period that is available to them before visitors arrive at their websites. This, it is argued, is the principal reason for the poor click and conversion rates the industry has become resigned to.

Imagine that the newspaper example could be applied to online advertising and that the data held about each web visitor could be processed using powerful decisioning algorithms to determine, before the website page they are on has even finished loading, whether to serve an ad and, if so, which ad to serve to that visitor. This would ensure that website visitors would only see ads appropriate to their behavioural profile. This would be achieved by comparing the profile of a new visitor with profiles of previously responsive customers and putting the bestperforming message in front of them, all in real time.

This approach is known in the industry as 'pre-view' advertising optimisation and it is designed to improve an advertiser's results significantly by delivering increased click-through, conversion and data acquisition by improving the relevance of advertising. It is based on the assumption that the more relevant the online advertising, the more satisfactory the customer experience and the greater the conversion ratio, resulting in enhanced customer loyalty and revenue.

\section{Online consumer behaviour - Understanding developing trends}

The manner in which consumers navigate the web is never static. Trends all to often emerge that surprise even the most experienced members of the industry as being counter-intuitive to what one would have reasonably expected. For example, what are the reasons that one group of consumers should be highly responsive to high-end credit card ads shown in the early hours of the morning, a group we will describe as 'late-late-night users' because they use the web between two and six o'clock in the morning, while a separate group of consumers with broadband connections are seemingly more flighty in their response to one brand over another? Is it simply because they can afford to be, given the speed of their connection, or are other there other, more interesting dynamics at play?

Understanding the reasons for differences in online behaviour of this

Understanding reasons for differences in online behaviour is important sort is of paramount importance to the long-term effectiveness of online marketing, whether in respect of search, banners, e-mail or the affiliate channel. So what sources of data can the online marketer get access to that could not be accessed by offline marketers and which will maximise the return of online marketing investment and improve the status of the medium (Figure 1)?

\section{Utilising behavioural targeting in online advertising}

This section reviews the various phases required in order to optimise the effectiveness of online advertising.

\section{Phase 1 - Planning for online advertising optimisation}

Behavioural targeting and online marketing optimisation are similar to any other marketing activity in that to be successful they need to be 


\section{Business rules must be taken into account}

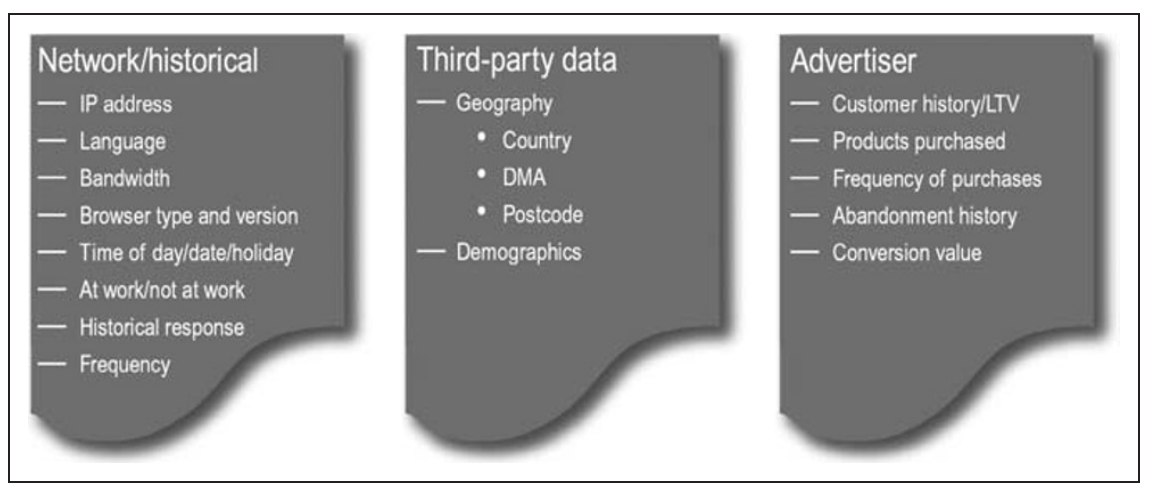

Figure 1: Sources of internet-specific behavioural data that can augment traditional offline sources of marketing data

driven by objectives. Objectives for online advertising can vary, so the focus of the campaign may be to drive more visits to a destination site or landing page (one would call this click maximisation) or more conversions (which is called conversion optimisation). Conversions can mean many different things — applications, downloads, purchases, signups etc.

But we may find our objectives and the delivery of the campaign 'constrained' by certain business rules that need to be taken into account - for example, if your campaign aggregates multiple products, the budget may come from different product owners who each want to ensure their product is shown according to the budget they have contributed, even if this is not considered optimal by the optimisation model. Or you could find a situation whereby one product is more profitable than another, and therefore if an online viewer is equally responsive to either product offer one would wish to show them the most profitable (profit maximisation). Whatever your campaign objectives are, these kind of considerations need to be made at the planning stage.

A key stage in the planning for optimisation is to 'architect' the decision-making process of the proposed campaign rules - that is, if online viewer X exhibits certain behavioural characteristics, then apply rule $\mathrm{Y}$ based on the assumption that $\mathrm{Y}$ is the most responsive creative or offer for viewer $\mathrm{X}$. Initially almost of all of these rules are likely to be subjective, though in time as a result of testing an increasing proportion of them will be founded on quantitative evidence.

An 'optimisation architecture', therefore, is a form of decision tree which maps out how the optimisation rules will treat viewers differently, depending on their behavioural characteristics (Figure 2).

\section{Phase 2 - Data acquisition}

In order to build a picture of online customer behaviour, some form of benchmark is required which measures the performance of an online system with no segmentation. Measuring the performance of an online segment against such a base, in other words one in which every visitor is treated in the same manner, makes it possible to identify exceptions to the 


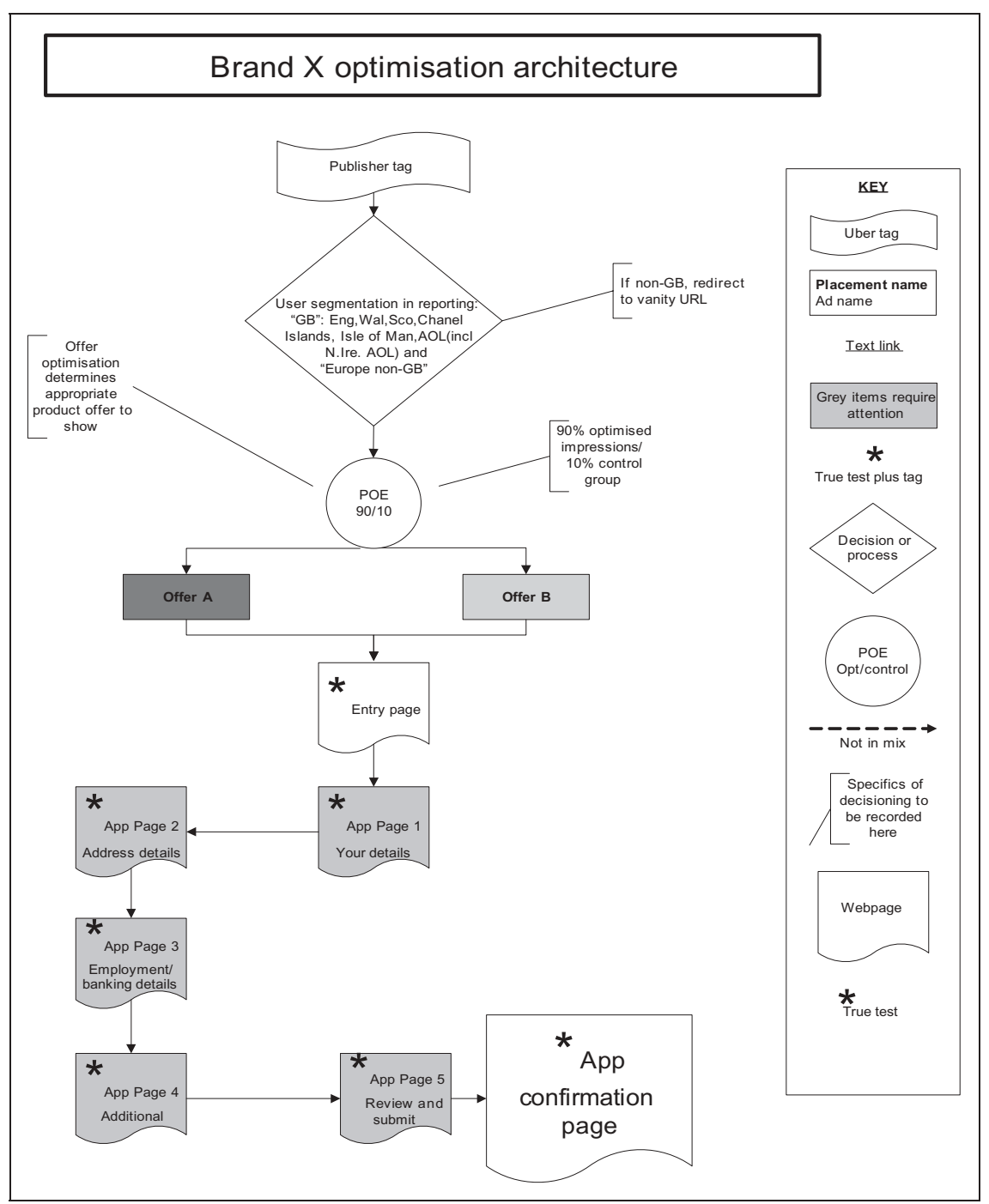

Figure 2: Example of optimisation architecture, showing how non-GB traffic is filtered out as non-responsive, and the ratio between optimised and control impressions

average pattern of behaviour and, ultimately, leads to decisioning strategies that will result in exceptions to the average pattern of response. To achieve this, it is prudent to decide upon the length of what might be called a 'data-collection' period (Figure 3). This is the period during which all the online ad impressions served to internet viewers are 'nonoptimised' (eg standard ad served) and during which any variations in the ad creative are randomly shown to every viewer. This forms a 'control' for the purpose of subsequent analysis.

The objective of the data-acquisition phase is to identify the 'clusters' (groups of internet viewers) that are most (or least) responsive to an advertiser's messages and to rank those groups according to their varying levels of responsiveness Thus, cluster 1 would include the most responsive group, representing the highest number of overall respondents from the total population of responders for that brand/campaign. 


\section{Media yield curve}

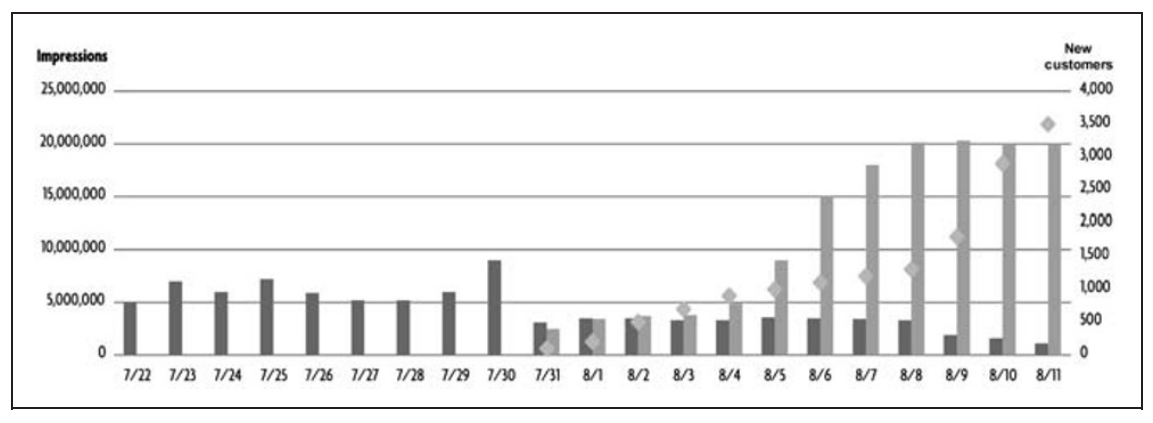

Figure 3: Illustration of a typical 'data-collection' period, initially representing a 100 per cent control group and then switching to 90 per cent optimised impressions and a 10 per cent control

Each cluster (customer group) is given a set of attributes against which they are deemed to be responsive. These attributes include anonymous internet-based data such as day part, geography and broadband or narrow bandwidth.

Once the data-collection phase has been completed, a 'media yield curve' (MYC) is created for the advertiser's campaign or product offer. An MYC is produced for each offer in the event that a multiple product offer campaign is being delivered (Figure 4).

The MYC is designed to represent two items of intelligence.

- It shows where the majority of an advertiser's response comes from within the total amount of media served. For example, it might show that 96 per cent of the response to the campaign for a particular credit card came from one or more advertisement strategies that represented only 58 per cent of the impressions on the media being served. This information helps the advertiser to identify and demonstrate where in the campaign a high degree of wastage in non-responsive media occurs.

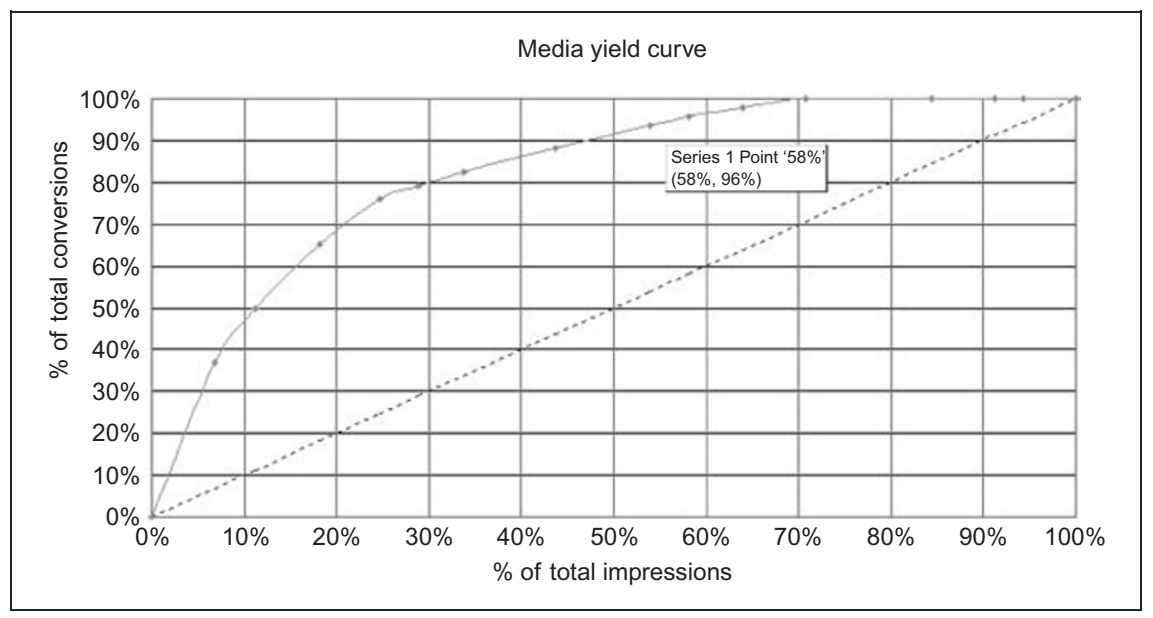

Figure 4: An MYC that plots where in the impressions delivery (horizontal axis), the response comes from (vertical axis); in the example shown, 96 per cent of response came from 58 per cent of media 
- The MYC will also show which of the clusters have been the most responsive. For example, if 76 per cent of overall credit card X applications came from one group of customers who all share common characteristics, this would group would form Cluster Rank 1.

In addition to the trend line shown in the MYC graph, data are also provided that identify important learnings such as which response attributes the cluster showed - eg geo, day part, exposure count and placement may have all featured in the clustering for that group.

Additionally, the MYC can show how much 'advertising' wastage there was in the data-collection period. That is, looking at the MYC in Figure 4 , if 96 per cent of conversions came from 58 per cent of media, to acquire another 4 per cent of conversions means serving another 42 per cent of media - clearly this is not optimal and the MYC data can help identify where this 'wastage' came from in terms of publishers and placements in the media plan, so that one can revise the media purchased going forward. The MYC, therefore, becomes an important planning tool.

\section{Placement optimisation}

\section{Phase 3-Optimisation}

Following completion of the data-collection period and analysis of the results, the next stage in the optimisation process involves building the optimisation model and the implementation of the decision-making rules needed to support the achievement of the required marketing objectives for the advertiser's campaign. This phase is often referred to as 'placement optimisation' and ensures that the right message, offer or creative is delivered to the right type of customer, at the right time and in the right placement.

For example, if the campaign objective is to maximise financial return on media spend, the model will determine which offers should be shown to which clusters to deliver the greatest profitability from that media investment. Thus, where an online viewer's behaviour matches the profile of two responsive clusters and is equally suited to more than one product offer, the model will only select the more profitable offer. Alternatively, if the objective is to build customer registrations, optimisation rules would be implemented which ensure that the most responsive offer, which may not necessarily be the most profitable one, will be shown to the most responsive cluster or clusters, ensuring a high number of registrations regardless of the profitability to the advertiser.

In order to assess the impact on a campaign of any optimisation and behavioural targeting strategy, an advertiser would typically hold back a control group of around 5-10 per cent of the non-optimised impressions from the overall impressions delivered. The precise size of this control group may vary according to the number of impressions that are needed by the optimisation model. It is against the performance of this control group that an advertiser can evaluate the performance of the "experiment group' and hence of the optimisation model.

In Figure 5 a cluster decision tree demonstrates how progressive optimisation creates increasingly more granular groups of customers, as respondents to an advertiser's messages differ in behaviour. It is this 


\section{Acting on data intelligently to influence purchasing behaviour}

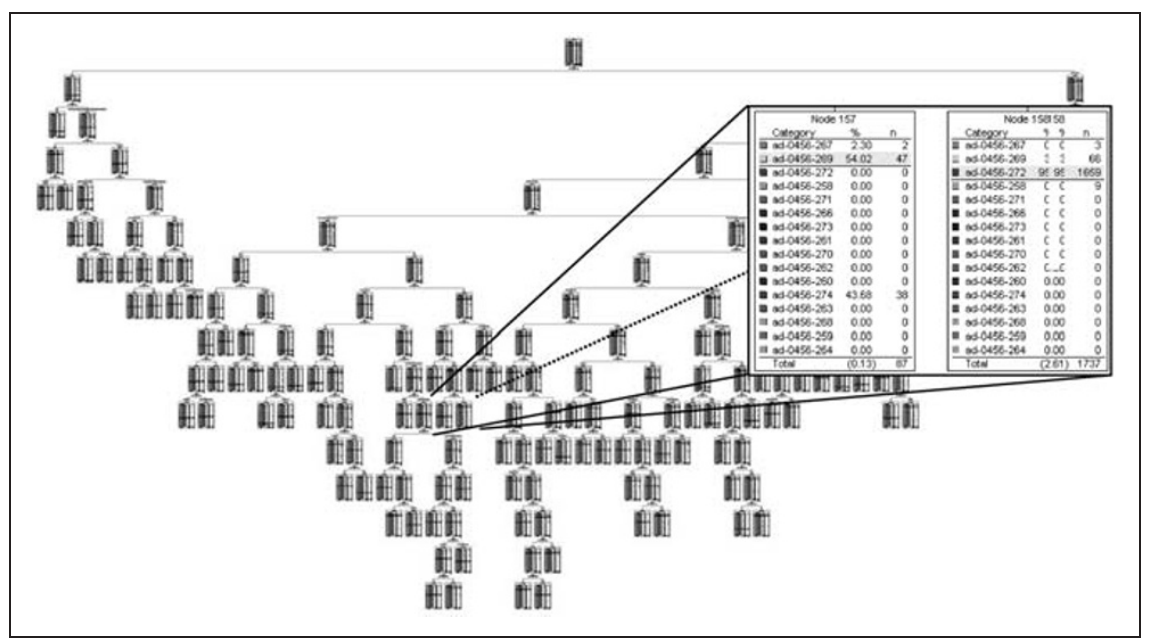

Figure 5: A 'cluster' decision tree showing how many differing groups of customers exist within an advertiser's campaign; each 'cluster' exhibits different response characteristics

decision tree that enables the optimisation model to deliver greater effective results, by matching a new viewer to one of the these previously responsive groups and then showing that new viewer the best-performing advertisement.

\section{Phase 4 - Results analysis}

The methodology typically applied to measuring online advertising effectiveness in today's market is to assess performance in terms of the score of a campaign on the following metrics:

- impressions delivered in relation to numbers purchased

- clicks achieved by offer/creative combinations (CTR)

- conversion totals and rates

— best-performing publisher/placement/creative combinations.

These metrics, while valuable from a 'click-stream' perspective, do not reveal sufficient information about a customer's behaviour, demographics or purchase decisioning online. For today's marketer, the challenge is not only meeting the need for richer data, but also acting on those data intelligently to influence the purchasing behaviour of viewers.

Behavioural targeting and optimisation add an extra dimension to the current approach, in the shape of 'cluster attributes' — that is, the data associated with the responsiveness and performance of different groups of customers exposed to the optimisation model.

Such cluster attributes include geography - that is, where in the UK or world are respondents' and converters' normal place of residence - or 'day part', the term used by the industry to describe the periods when customers are most or least responsive to a particular ad.

Additionally, using behavioural targeting, an advertiser or an advertiser's media agency can tell which impressions were served to customers who were not responsive. This segment is referred to as a 
'reject' group, and provides useful information on the demographics of viewers on whom ad impressions, and therefore money, are currently being wasted. It is this wastage that affects overall CpA (cost per acquisition) and ROI performance. Removing this wastage is one of the significant improvements to the profitability of online advertising that behavioural targeting can deliver.

The key to removing this 'wastage' is a process often described as 'media optimisation', which involves an agreement between the advertiser and the publishers to perform a 'passback' process for an advertiser's campaign. This means that if the profile of a viewer in a placement does not fit a responsive cluster, the advertiser's ad is not served, resulting in the impression not being used and the cost not being incurred. This approach reduces the overall wastage typically experienced in online advertising and increases the effectiveness of media.

A softer approach to passback, which does not require publisher involvement, is what is called 'pass-sideways', where in the same event of a viewer not fitting an advertiser's responsive clusters, rather than passback the impression altogether, it is still served but another advertiser's message is shown, for which that viewer is deemed more responsive. This means an advertiser's campaign can operate much more like a network, where one simply lets the modelling determine which advertiser to show to the viewer.

This is a radically different approach to traditional online media planning and buying, and one that has been widely accepted in the USA by both publisher and advertiser communities because both parties benefit from greater productivity from the inventory they are buying/selling.

\section{Phase 5 - Going beyond the CTR}

Behavioural targeting and online marketing optimisation can help identify key trends in any business, ranging from seasonality to problems with website sales processes. By linking viewer/respondent behaviour directly to the sales funnel data, it is possible to establish which customers are ultimately most likely to purchase and which are most likely to drop out. This enables the advertiser to treat them more appropriately, thereby ensuring that there are improvements not just in CTR but in conversion rates also.

A consistent problem with measuring online advertising's contribution is that there is no benefit in driving traffic to a website landing page if 80 per cent of arrivals at the landing page then drop off. Behavioural targeting can start to identify why respondents dropped out. For some groups it is possible that the sales process was too cumbersome perhaps as a result of asking for too much information at too early a stage or before the viewer had a commitment to purchase, a trend typical of many broadband customers.

Another trend that often rewards analysis is timing. Figure 6 shows how the registration rate of a campaign declines in line with impression delivery. This demonstrates that, regardless of the channel customers come from (search, affiliate or advertising), if the timing of the campaign is not right this will directly affect conversion. 
Critical role of sales funnel analysis

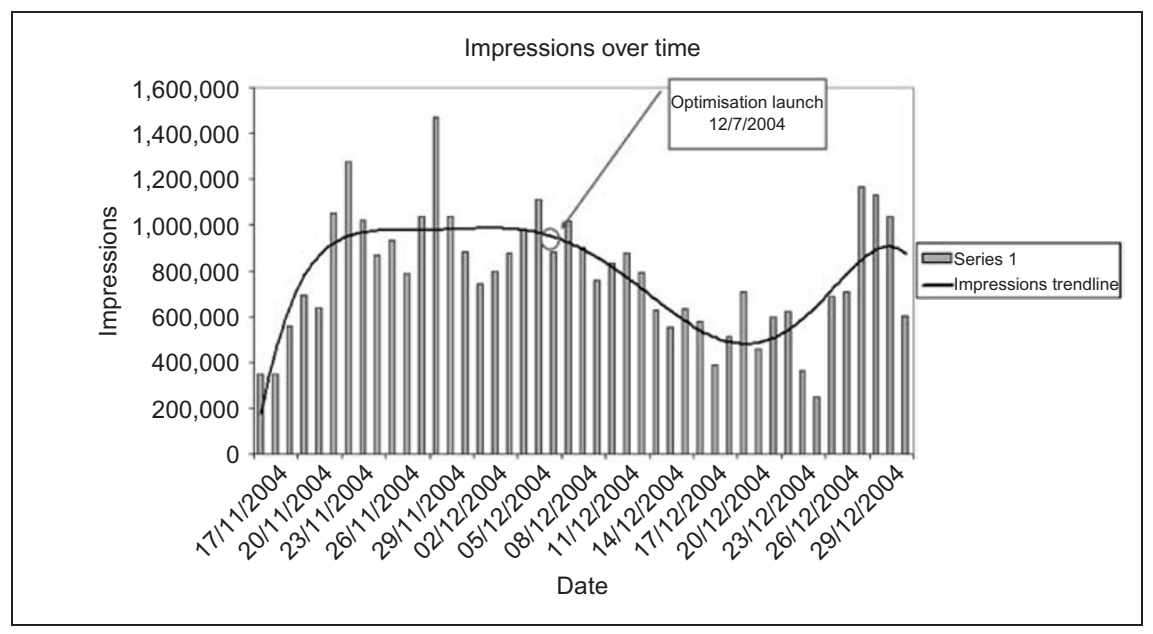

Figure 6: Impression delivery over campaign duration

Clearly, where there is a dip in response, due to seasonality, offer or some other factor affecting the click trend curve, there will also be a direct effect on conversion (see Figures 7 and 8).

Sales funnel analysis has a critical role in ensuring that the benefits of behavioural targeting in identifying and attracting the 'right type' of customers to your website landing page are reinforced by decision strategies that maximise sales conversion. Figure 9 shows the 'sales funnel' in diagrammatic form. Such a diagram can be drawn in the event that each page in the sales process of a website is 'tagged' with a tracking tag that records clicks and conversions from the online advertising campaigns.

Using these advertiser-side tracking tags, it is then possible to begin to identify where in the sales process 'drop out' is occurring and identify ways in which one can fix the problem.

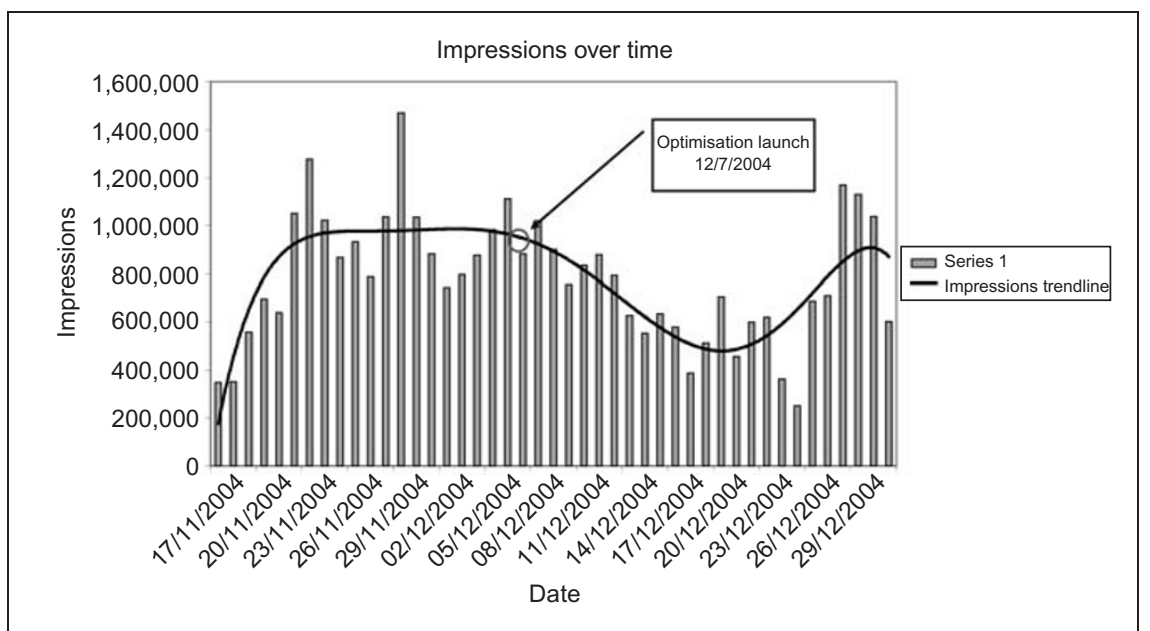

Figure 7: Registration trend from all sources over campaign duration 


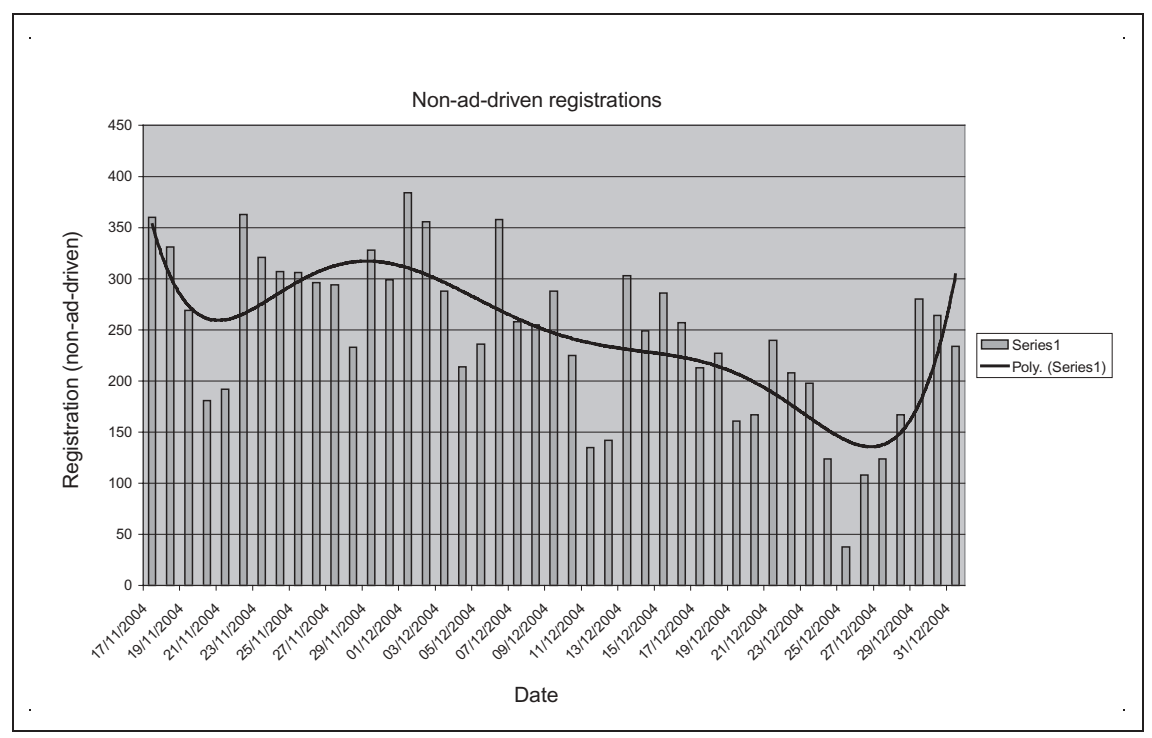

Figure 8: Registration trend from ad source over campaign duration

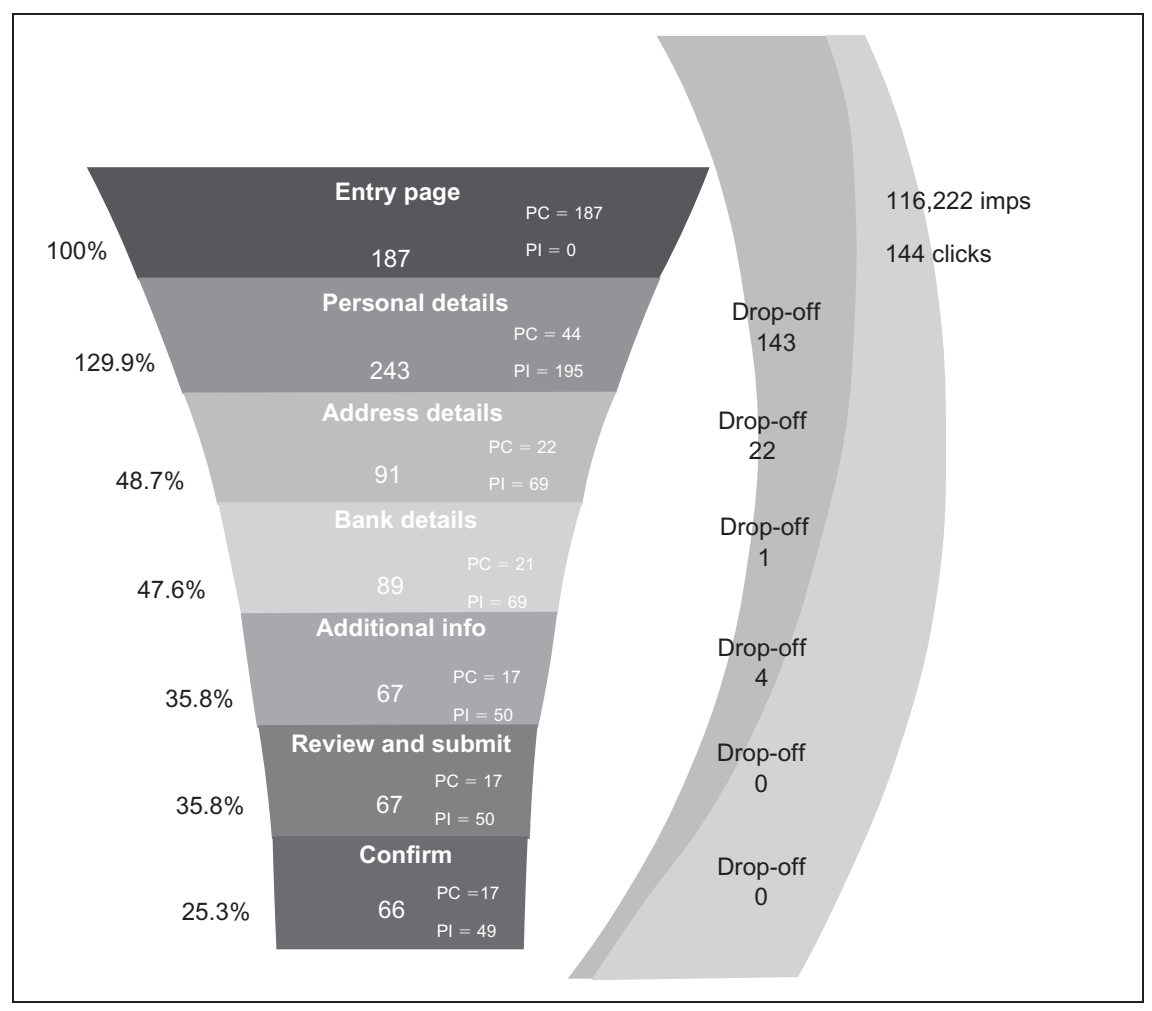

Figure 9: Sales funnel analysis

\section{Recommendations for effective online advertising}

Behavioural targeting and online advertising optimisation can deliver a great number of insights into how an advertiser's planned media can perform.

By implementing campaign activity tests the effectiveness of 
optimisation under a variety of different conditions and different elements of the campaign can be tested, and the optimal mix can be quickly found.

\section{Recommendations}

- The offer matrix - Enables the advertiser to present a product set that contains suitably differentiated offers. This should ensure that the decisioning model has enough data to calculate which clusters are going to be responsive to which product offers. Products that are not suitably differentiated will not stand to gain as much lift in click and conversion as products that are highly different.

- The creative matrix - Does not use a limited set of messages, but offers a broad selection of product creatives. This enables optimisation to deliver more clusters and, therefore, more effective optimisation results.

- The publisher matrix - Media booking timescales may often result in restricted access to the best-performing media across the publishers used in a campaign. For example, financial services targeted media is in high demand and at a premium cost, running as high as $£ 70 \mathrm{CpM}$. An increase in timescale to plan and book media will enable a business to secure access to better-performing media.

- Media planning - Agree strategic campaign and performance objectives for each product and offer, which will provide the information required to produce the creative brief based on the right media schedule.

- Publisher partnerships - Identify key partner publishers for a campaign. These can be identified from the MYC by identifying which publishers and placements are performing best and which are non-responsive. Look for where your best-performing clusters come from and leverage this opportunity.

- Media optimisation - Negotiate with publishers the inclusion of media optimisation (passback) to all campaigns based on 'reject group' analysis. Or, at the very minimum, consider alternative ways of managing the reject group effectively, by using the pass-sideways approach for example.

- Sales funnel - Review your sales funnel and identify reasons for drop off occurring at each stage, with a view to identifying trends across all channels, including search and affiliate. Gain an understanding of the key differences of respondent profiles with a view to creating 'application scenes' that match a user's behaviour. This will help reduce conversion barriers and, ultimately, drop off.

- Reporting and analysis - Develop bespoke reporting and analysis formats, highlighting key data requirements that seek to maximise your learnings from online planned media strategy.

- Progressive modelling - Monitor your advertising optimisation model on a continual real-time basis, reassessing its performance daily or weekly and adapting the model by implementing appropriate changes to maximise the value of activity.

- DM offline strategy - Review offline offers available to demonstrate how online ad optimisation can apply similar messaging and deliver segmentation, using clustering data. Agree a long-term strategy that 
will fully integrate offline DM and online optimisation to create a seamless customer experience across those channels.

\section{In summary}

Behavioural targeting and real-time decisioning are changing the way we plan, buy and serve online advertising campaigns. What this methodology does for advertisers is, in real time, reduce the wastage in their campaigns, improve the effectiveness of targeting and ultimately deliver a greater click and conversion rate, which, in turn, improves ROI.

(C) James A. Matthewson 